\title{
Management of tendon haemangiosarcoma in a Bactrian camel (Camelus bactrianus) - a case report
}

\author{
Pavel Kvapil ${ }^{1,3}$, Marjan Kastelić1 ${ }^{1}$ Bojana Stranjać ${ }^{1}$, Mitja Gombač², Tanja Svara², \\ Eva Bártová ${ }^{3}$, Matej Drobnić ${ }^{4}$
}

${ }^{1}$ ZOO Ljubljana, Slovenia

${ }^{2}$ University of Ljubljana, Veterinary Faculty, Institute of Pathology, Wildlife, Fish and Bees, Ljubljana, Slovenia

${ }^{3}$ University of Veterinary and Pharmaceutical Sciences Brno, Faculty of Veterinary Hygiene and Ecology, Department of Biology and Wildlife Diseases, Brno, Czech Republic

${ }^{4}$ University Medical Centre Ljubljana, Department of Orthopedic Surgery, Ljubljana, Slovenia

Received November 30, 2016

Accepted March 27, 2017

\begin{abstract}
An 18-year old intact female Bactrian camel (Camelus bactrianus) was suffering from lameness due to a mass on the right dorsal metacarpal region that caused acute swelling and local skin necrosis. Histology examination and immunohistochemistry of the biopsy material of a mass revealed haemangiosarcoma of the extensor tendons. Three weeks after the biopsy, the tumour was enlarged to $6 \mathrm{~cm}$ in diameter and the animal became disabled. The tumour with its associated tendon were resected and the tendon's edges were bridged with a synthetic polytape graft. The camel was fully weight-bearing after the surgery. Two weeks later, the graft was removed due to widespread necrosis. Since the wound was positive for Corynebacterium sp., Acinetobacter iwoffi, Micrococcus sp., Escherichia coli, and Staphylococcus sp., the post-operative antibiotic treatment was prolonged for 28 days. Four months later, the wound healed using daily irrigation and bandaging and the camel walked normally. Nine months after diagnosis, the camel suddenly died without any clinical signs. Metastases of the haemangiosarcoma were found in the liver, lungs, kidneys, brain, meninges, and mediastinum. Exsanguination due to rupture of a liver metastasis was determined as the cause of the death. Haemangiosarcoma is a malignant neoplasm that arises from endothelial cells of blood vessels and tends to be very aggressive. To the author's knowledge, this is the first case report of a metastasizing haemangiosarcoma arising from the lateral extensor tendon in a Bactrian camel.
\end{abstract}

Camelids, polytape graft, metastases, neoplasia, tendon surgery

There are only few reports on neoplasia in Old World camelids compared to llamas or alpacas (Effron et al. 1977; Valentine and Martin 2007; Molenaar et al. 2009). In Bactrian camels (Camelus bactrianus), metastasing gastric adenocarcinoma, histiocytic sarcoma, meningioma, and a vaginal leiomyoma have been reported to date (Molenaar et al. 2009). In the Arabian dromedary (Camelus dromedarius), an ovarian teratoma, bronchoalveolar adenocarcinoma, lymphatic leukaemia, renal cell carcinoma, ulnar osteosarcoma, pulmonary leiomyoma, squamous cell carcinoma, fibroma, lipoma, and fibromyxosarcoma have been described (Molenaar et al. 2009; Fahd and Yasmin 2013; Gamal and Shawky 2013). To our knowledge, neoplasia involving the tendons has not been reported in camelids yet.

\section{Materials and Methods}

An 18-year old female Bactrian camel from ZOO Ljubljana was examined due to the lameness of the right thoracic limb. The animal suffered from a solid movable circular mass (measuring $4 \mathrm{~cm}$ in diameter) without ulcerations on the right dorsal metacarpal region. The camel was put under general anaesthesia during incisional biopsy of the lesion. It was determined that the mass was in contact with the extensor tendon and seemed to be

Address for correspondence:

Doc. MVDr. Eva Bártová, Ph.D.

Department of Biology and Wildlife Diseases

Faculty of Veterinary Hygiene and Ecology

University of Veterinary and Pharmaceutical Sciences Brno

Palackého tř. 1946/1, Brno 612 42, Czech Republic 
growing around the tendon. Biopsy material was fixed in $10 \%$ buffered formalin, embedded in paraffin, sectioned at $4 \mu \mathrm{m}$, and stained with haematoxylin and eosin. Histology examination revealed a poorly demarcated, unencapsulated neoplastic mass, composed of infiltrative, pleomorphic, spindle, ovoid or oval shaped, anisocytotic neoplastic cells with prominent nucleoli, which formed irregular blood-filled clefts, channels and cavities. Bi- or even multinucleated cells were seen. Eighteen mitotic figures were counted per 10 high power fields. Large, multifocal areas of haemorrhage and necrosis with neutrophil infiltration were scattered throughout the tumour. Immunohistochemical assay was done using the mouse monoclonal anti-von Willebrand factor antibody (DAKO, Denmark) for the immunolabelling of antigen.

The sections of specimen were incubated with primary antibodies for one hour at room temperature in a humid chamber. Peroxidase activity was stopped with Peroxidase-Blocking Solution (DAKO, Denmark) for $30 \mathrm{~min}$ at room temperature. Subsequently, visualization kit (DAKO REAL ${ }^{\mathrm{TM}}$ EnVision $^{\mathrm{TM}}$ Detection System Peroxidase/DAB+, Rabbit/Mouse, Denmark) was used according to the manufacturer's instructions. Slides were counterstained with Mayer's haematoxylin and mounted. Positive (normal endothelium) and negative (incubation with antibody diluent (DAKO Denmark) without the primary antibody) controls were included. Neoplastic endothelial cells were diffusely and strongly positive for von Willebrand factor (Plate VI, Fig.. 1). Based on histopathological and immunohistochemical findings, the tumour was diagnosed as haemangiosarcoma.

\section{Results}

Three weeks after the biopsy, the mass enlarged to $6 \mathrm{~cm}$ in diameter and the animal became disabled and therefore, put again under general anaesthesia. Pre-operative blood analysis revealed normal haematological and biochemical values. The tumour was dissected from the surrounding tissue together with an $8 \mathrm{~cm}$ section of the tendon (Plate VI, Fig. 2). The edges of the tendon were bridged with one doubled synthetic graft of Neoligaments Polytape $40 \times 800$ (Xiros, United Kingdom). Polytape was fixed to the tendon stumps with a modified Kessler suture under appropriate tension to preserve the original tendon length. Bacteriological examination of the sample from the surgical site was negative. Despite loss of the surrounding skin due to necrosis, the surgical wound was closed and the graft was entirely covered with the skin. A soft bandage was applied to the lower leg. Perioperative and postoperative antibiotic prophylaxis was initiated with enrofloxacin $(5 \mathrm{mg} / \mathrm{kg}$ i.m., once daily = s.i.d. Enroxil $100 \mathrm{mg} / \mathrm{ml})(\mathrm{Krka}$, Slovenia). Results of post-anaesthesia haematology and biochemistry were unremarkable. Sixth day after surgery, the camel was able to walk short distances without limping. The wound was positive for Corynebacterium sp., Acinetobacter iwoffii, Micrococcus sp., Escherichia coli, and Staphylococcus sp. which is why the post-operative antibiotic treatment (enrofloxacin) was prolonged for further 28 days. Three weeks after surgery, the synthetic polytape graft was the only remaining bridging structure, and there were no means to retain the graft in spite of daily irrigation and debridement (Plate VII, Fig. 3). Because the tendon stumps necrotized, the graft was removed one month after surgery. The wound started to heal due to daily irrigation and bandaging. Four months later, the wound healed completely and the camel was able to walk normally. However, the animal suddenly died nine months after the first examination and was immediately submitted for necropsy.

The camel was in good body condition, but anaemic. Twelve litres of blood and nine litres of clotted blood were found in the abdominal cavity. Numerous reddish-black, rounded, smooth, well-demarcated, bulged, firm neoplastic nodules up to $6 \mathrm{~cm}$ in diameter were scattered throughout the liver parenchyma, lungs, kidneys, brain, meninges, and mediastinum. One of the neoplastic nodules in the quadrate hepatic lobe, of $4 \mathrm{~cm}$ in diameter, was ruptured. All heart chambers were severely dilated. No gross evidence of tumour recurrence was observed at the tumour excision site. Samples of hepatic, pulmonary, renal, mediastinal, and cerebral nodules, in addition to heart, spleen, pancreas, stomach, intestine, spinal medulla, and soft tissues from the location of tumour excision were collected for histology analysis. The metastatic nodules were noted to be poorly demarcated and unencapsulated, and were composed of cells similar to those seen in the primary tumour of the lateral toe extensor tendon. In contrast to the primary tumour, the 
mitotic index was higher (62 mitoses per 10 high power fields counted). These tumours were diagnosed as metastatic haemangiosarcoma of the liver, lungs, kidneys, mediastinum, brain, and meninges. No neoplastic changes were microscopically observed in other examined organs and tissues or in the surgical site.

\section{Discussion}

Haemangiosarcoma is a malignant neoplasm that arises from endothelial cells of blood vessels (Robinson and Robinson 2016). It is an aggressive vascular tumour with a high malignant potential (Ravi and Patel 2013). It has been mostly described in dogs involving the spleen, right atrium, skin, subcutis, and liver (Withrow et al. 2013), but there was only one report of haemangiosarcoma in the gastrocnemius tendon in a dog (Waren and Summers 2007). To our knowledge, the only reported case of haemangiosarcoma in the camelids was an intraosseous haemangiosarcoma in a three-month old llama that metastasized into lungs and lymph nodes (Hamir et al. 1997).

This kind of tumour tends to have a very aggressive biological behaviour commonly with rapid and widespread metastases (Withrow et al. 2013), therefore requiring multimodality care for optimal outcomes. The efficiency of preoperative radiotherapy or chemotherapy to increase the survival rate and to prevent metastases in animals remains unknown. These tumours tend to be multifocal, and systemic therapy in the neoadjuvant setting is strongly recommended even for a localized disease. Effective cytotoxic chemotherapy is available for metastatic angiosarcomas, but their durability is limited (Ravi and Patel 2013).

In the current case report, we presume that the primary tumour location was in the extensor tendon since haemangiosarcoma metastasizing to a tendon is less likely and the survival time as well as metastatic mass numbers and size were significantly small regarding the duration of the period after tumour excision. Surgical reconstruction with a graft was indicated, because severe walking difficulties and a toe drop due to the extensor tendon involvement were expected. Post-operative bracing is not possible in the camelids and biological reconstruction was expected to fail. The synthetic graft allowed immediate full weight-bearing of the affected limb. Complications during the wound healing process were probably initiated by marginal skin necrosis as the skin was previously compromised by tumour, and by a secondary infection. The primary incompatibility of the synthetic graft is less probable since this graft has been used in human medicine for over two decades in ligament and tendon reconstructions (Zaffagnini et al. 2008; Chen et al. 2009). Nevertheless, at the end of the treatment, the camel was able to walk normally even without the reconstructed tendon. Therefore, it seems that loss of the lateral toe extensor tendon can be sufficiently compensated for by other muscle groups, at least in camels living in a zoo setting. Clinical signs are usually related to the site of origin of the primary tumour or to the presence of metastases, spontaneous tumour rupture, coagulopathies, or cardiac arrhythmias (Nelson et al. 2005). Nevertheless, the animal did not show any clinical signs (until a few hours prior to death), despite the presence of widespread metastases in several organs. In dogs with splenic or cardiac haemangiosarcoma, some episodes of collapse are common as a result of ventricular arrhythmias (Effron et al. 1977; Nelson et al. 2005). Prognosis of cases where metastases are present are poor without systemic chemotherapy, whereas in the absence of metastases it could even be excellent (Withrow et al. 2013). To the author's knowledge, this is the first case report of a metastasizing haemangiosarcoma arising from the lateral extensor tendon in a Bactrian camel that was successfully addressed surgically. Surgical removal of tumours on tendons and subsequent reconstruction with a synthetic graft seems to be a promising solution that needs to be further investigated. 


\section{References}

Chen J, Xu J, Wang A, Zheng M 2009: Scaffolds for tendon and ligament repair: review of the efficacy of commercial products. Exp Rev Med Devic 6: 61-73

Efron M, Griner L, Benirschke K 1977: Nature and rate of neoplasia found in captive wild mammals, birds and reptiles at necropsy. J Natl Cancer Inst 59: 185-198

Fahd AAS, Yasmin OEA 2013: Throughout pathological study on skin, subcutaneous and mucosal neoplasia of the dromedary camel. Braz J Vet Pathol 6: 48-55

Gamal W, Shawky AM 2013: Pulmonary leiomyoma in a dromedary camel (Camelus dromedarius). J Vet Med: Res Rep ID 773813

Hamir AN, Pierce V, Richardson D 1997: Intraosseous hemangiosarcoma with metastasis in a three-month-old llama. J Vet Diagn Invest 9: 210-213

Molenaar FM, Breed AC, Flach EJ, McCandlish IAP, Pocknell AM, Strike T, Routh A, Taema M, Summers BA 2009: Brain tumours in two Bactrian camels a histiocytic sarcoma and meningioma. Vet Rec 164: 684-688

Nelson WR, Guillermo CC 2005: Manual of Small Animal Internal Medicine. $4^{\text {th }}$ edn. St. Louis, Missouri: Elsevier Mosby

Ravi V, Patel S 2013: Vascular sarcomas. Curr Oncol Rep 15: 347-355

Robinson WF, Robinson NA 2016: The cardiovascular system. In: Maxie MG (Ed): Jubb, Kennedy, and Palmer's Pathology of Domestic Animals, vol. 3. $6^{\text {th }}$ edn. San Diego, CA, USA: Academic Press, pp 2-101

Valentine BA, Martin JM 2007: Prevalence of neoplasia in llamas and alpacas. J Vet Diagn Invest 19: 202-204

Warren AL, Summers, BA 2007: Epithelioid variant of hemangioma and hemangiosarcoma in the dog, horse, and cow. Vet Pathol 44: 15-24

Specific Malignancies in the Small Animal Patient. In: Withrow SJ, Vail DM, Page RL (Eds) 2013: Withrow and MacEwen's Small Animal Clinical Oncology. $5^{\text {th }}$ edn. St. Louis, Missouri: Elsevier Saunders

Zaffagnini S, Marcheggiani MGM, Chatrath V, Bondi A, De Pasquale V, Martini D, Bacchelli B, Marcacci M 2008: Histological and ultrastructural evaluation of Leeds-Keio ligament 20 years after implant: a case report. Knee Surg Sports Traumatol Arthrosc 16: 1026-1029 
Plate VI

Kvapil P. et al.: Management of tendon ... pp. 97-100

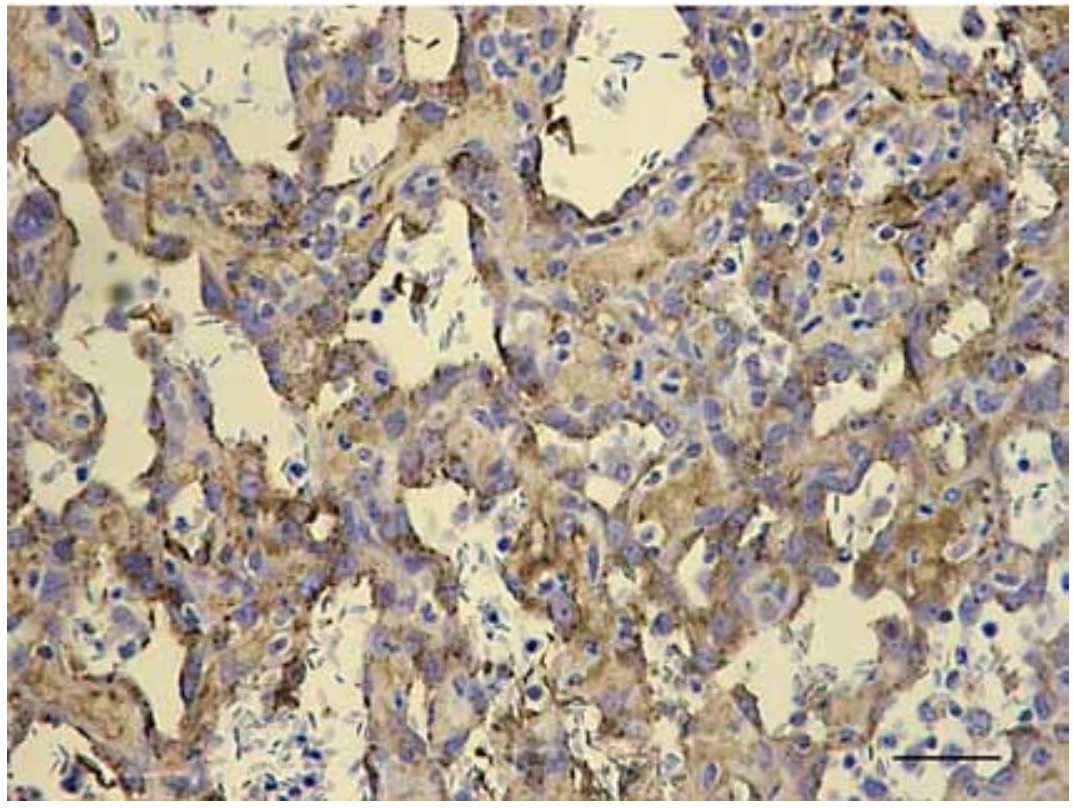

Fig. 1. Haemangiosarcoma in a Bactrian camel with neoplastic endothelial cells strongly positive for von Willebrand factor. Immunohistochemistry, $\times 400$, bar $=500 \mu \mathrm{m}$

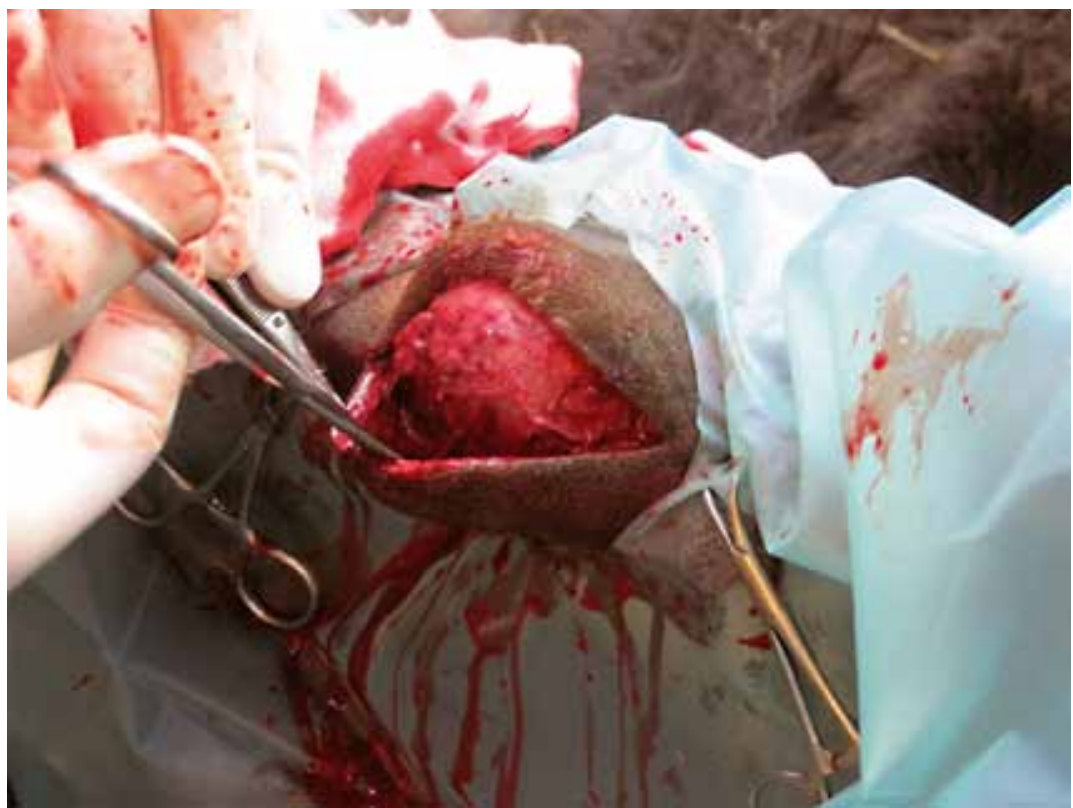

Fig. 2. Surgical excision of a tumour on the right dorsal metacarpal region in a Bactrian camel. 


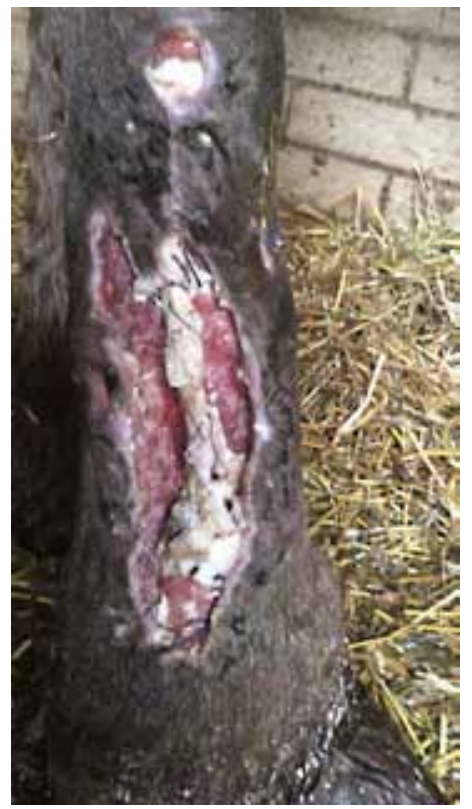

Fig. 3. Synthetic polytape graft in the necrotic wound after one month of treatment in a Bactrian camel. 